\title{
"É NATURAL, É BOM! SÃO FRUTOS QUE VEM DA NATUREZA": REPRESENTAÇÕES LOCAIS SOBRE O CONSUMO DE PLANTAS ALIMENTÍCIAS SILVESTRES EM UMA ÁREA RURAL DO BRASIL
}

\author{
"IT'S NATURAL, IT'S GOOD! THEY ARE FRUITS THAT COME FROM NATURE": \\ LOCAL REPRESENTATIONS ABOUT CONSUME OF WILD FOOD PLANTS IN A \\ RURAL POPULATION OF BRAZIL
}

Juliane Souza Luiz HORA ${ }^{1 *}$; Taline Cristina DA SILVA²; Viviany Teixeira do NASCIMENTO³

\begin{abstract}
${ }^{1}$ Programa de Pós-graduação em Etnobiologia e Conservação da Natureza, Universidade Federal Rural de Pernambuco, Brasil. *jsl.hora1@gmail.com; ${ }^{2}$ Universidade Estadual de Alagoas, Brasil talinecs@gmail.com; ${ }^{3}$ Universidade do Estado da Bahia, Departamento de Ciências Humanas (DCH, campus IX), Bahia, Brasil. vivyteixeira@gmail.com
\end{abstract}

\section{RESUMO}

Submitted.23/03/2020; Accepted: 29/05/2020

A dieta de populações humanas em todo o mundo tem passado por mudanças substanciais, ocasionadas por forças não dirigidas provenientes de mudanças tanto no clima e no tempo, como também mudanças no mercado. Tais modificações diminuem a diversidade de alimentos na dieta das pessoas, em especial, nos países em desenvolvimento. Assim, substituem-se alimentos tradicionalmente coletados em ambientes naturais por outros de origem industrial, gerando preocupações sobre sua segurança alimentar. Este estudo realizou uma avaliação qualitativa das representações das pessoas sobre os fatores que podem impulsionar ou frear o consumo de dez plantas alimentícias silvestres mais conhecidas e utilizadas em uma região de semiárida no nordeste do Brasil. O consumo das espécies foi relacionado a representações de benefícios à saúde, geração de sentimentos de bem-estar e cura. Além de relações entre fatores ecológicos e econômicos como, a disponibilidade das espécies no ambiente e a substituição por produtos industrializados, sendo percebidos como limitantes para o consumo. Contudo, mesmo com o consumo descontínuo traz evidência de que plantas alimentícias silvestres continuam fazendo parte da dieta local, sendo um indício dessa permanência em contextos rurais e sua importância, em contraposição a maior parte dos achados que mostram o efeito inverso.

Palavras-chave: Cerrado, Discurso do Sujeito Coletivo, Etnobotânica, Plantas Alimentícias, Representação

\begin{abstract}
The diet of human populations around the world has undergone substantial changes, caused by undirected forces arising from changes in both climate and weather, as well as changes in the market. Such changes reduce the diversity of foods in the diet of human populations, especially in developing countries, replacing foods traditionally collected in natural environments with foods of industrial origin, raising concerns about their food security. This study carried out a qualitative assessment of people's representations of the factors that can boost or curb the consumption of ten best-known and used wild food plants in a semi-arid region in northeastern Brazil. The consumption of the species was related to representations of health benefits, generation of feelings of well-be and healing. In addition to relationships between ecological and economic factors, such as the availability of species and the replacement by industrializes products, perceived as limiting to consumption. However, even with discontinuous consumption it brings evidence that wild food plants continue to be part of the local diet, being an indication of this permanence in rural contexts and its importance, in contrast to most of the findings that show the opposite effect.
\end{abstract}

Key words: Cerrado, Collective Subject Discourse, Ethnobotanic, Food Plants, Representation 


\section{INTRODUÇÃO}

A dieta de populações humanas em todo o mundo tem passado por mudanças substanciais, ocasionadas por forças não dirigidas geradas por mudanças no clima e no tempo; forças econômicas e industriais (como a mudança de mercado); migrações e urbanização, e aumento da indústria agrícola, criando mudanças nos tipos e quantidades de alimentos disponíveis (Kuhnlein \& Receveur 1996). Em contra partida países industrializados, possuem uma maior disponibilidade de alimentos diversos devido ao aumento das técnicas do setor agroindustrial, no entanto, tal diversidade não corresponde à segurança alimentar de toda a população, as diferenças econômicas e sociais influenciam na compra de alimentos de baixa energia, densos e de má qualidade por terem um menor custo monetário (Nyambayo, 2015).

Em áreas rurais de países em desenvolvimento, por exemplo, pode-se observar um cenário similar, sendo provável que tenham uma parcela crescente da demanda urbana por alimentos importados (Satterthwaite et al. 2010). No entanto, mesmo com tais mudanças existe uma forte relação entre as populações humanas e os recursos naturais, principalmente, em regiões semiáridas brasileiras, em que espécies silvestres tem o seu uso alimentício reconhecido, apesar disso muitas destas plantas são subutilizadas devido a diversos fatores sociais e ambientais, omitindo os seus papéis na segurança alimentar (Nascimento et al. 2011; Nunes et al. 2018).

Plantas silvestres são tratadas nesse estudo como plantas não cultivas que podem ser coletadas em ambientes naturais (incluindo ao redor das residências, rios, lagos, florestas, e até mesmo paisagens agrícolas) (Cotton, 1996; Bhrarucha \& Pretty, 2010). Estas plantas consideradas alimentícias de regiões semiáridas possuem um elevado potencial nutricional, podem e devem ser utilizadas em programas para melhorar a qualidade alimentar de populações rurais que vivem nestas regiões (Nascimento et al. 2011). A diminuição de alimentos na dieta devido ao baixo consumo de alimentos silvestres, pode gerar implicações nutricionais, uma vez que esses recursos fornecem micronutrientes, como vitamina A, ferro, folato, niacina e cálcio (Ickowitz et al. 2016). Estes vegetais não fazem parte da estrutura dietética destas populações como, por exemplo, o milho e o feijão (Arnold et al. 2011), assim, o seu consumo pode aumentar a qualidade alimentar, ao que passo que aumenta a variedade já disponível pela produção agrícola de larga escala (Hall et al. 2019).

Um estudo realizado por Nascimento et al. (2015) em uma área semiárida de savana no nordeste do Brasil identificou 36 espécies de plantas silvestres conhecidas como recursos alimentares na região, das quais todas fazem parte da dieta local da população. No geral, as pessoas consomem os frutos dessas espécies, principalmente, in natura ou na forma de sucos ou doces. Além disso, algumas espécies fornecem óleos, que podem ser utilizados em diferentes receitas e para fins medicinais (Nascimento et al. 2015). Esse cenário de grande importância das plantas alimentícias nativas da região nos motivou a realizar um estudo com o objetivo de compreender as motivações que influenciam a continuidade do uso desses recursos, em contraposição a maior parte dos achados que mostram uma diminuição no consumo de plantas nativas.

Portanto, é fundamental entender as motivações e tendências que estão associadas ao abandono ou permanência do consumo de plantas alimentícias silvestres. Assim, o presente estudo foi desenvolvido em uma área rural no nordeste do Brasil, com um ecossistema de domínio Cerrado, na mesma área em que Nascimento et al. (2015) realizaram o seu estudo. Com principal objetivo de entender os fatores ligados a processos de escolha e permanência do consumo de plantas alimentícias silvestres, por meio da avaliação das representações das pessoas sobre estes recursos alimentícios.

\section{MATERIAL E MÉTODOS \\ Área de estudo}

Este estudo foi realizado com moradores da comunidade rural do Vau da Boa Esperança (Figura 1), pertencentes a um distrito com o mesmo nome, localizado na cidade de Barreiras ( $12^{\circ} 12^{\prime} 07,87^{\prime \prime S}$, $45^{\circ} 13$ '29, 29 "W), extremo oeste do estado da Bahia, nordeste do Brasil, de agosto de 2014 a março de 2015. Barreiras possui clima tropical (Aw / Köppen-Geiger) (Peel et al. 2007) e duas estações 
definidas a estação seca de maio a setembro e estação chuvosa de outubro a abril. A precipitação anual variando de 800 a $1.600 \mathrm{~mm}$ e as temperaturas mínima e máxima variam de $20^{\circ} \mathrm{C}$ a $26^{\circ}$, respectivamente (Batistella et al. 2002).

A cidade de Barreiras possui aproximadamente uma população de 155,439 habitantes (IBGE, 2019), 11\% dessa população pertence a áreas rurais que circundam o centro urbano. A região do município está inserida num domínio de Cerrado, sendo caracterizado por um mosaico complexo de vegetação, possuindo formações do tipo campo limpo, apresentando o predomínio de gramíneas, campo sujo, uma savana com características arbustivas e sensu stricto, bosque e cerradão, considerado uma floresta alta (Oliveira \& Marques, 2002). O Cerrado também apresenta um elevado número de grupos biológicos endêmicos (Brandon et al. 2005). Além disso, a região de Barreiras tem passado por intensivas transformações e mudanças na paisagem, ocasionadas ao uso e ocupação das terras, direcionado pelo setor agroindustrial fazendo dela um dos principais produtores nacionais de grãos, carnes, frutos e fibras (Mendonça, 2006; Queiroz et al. 2017).

A comunidade alvo de estudo está inserida dentro desse mosaico de formações vegetativas, situada dentro de um vale, dista cerca de $32 \mathrm{~km}$ do centro urbano. Sua população é composta por 14 famílias e caracterizada por indivíduos com idades maiores a 46 anos. O primeiro passo foi entrar em contato com representantes da comunidade para discutir os objetivos e propostas deste estudo e o desenvolvimento do Termo de Consentimento Livre e Esclarecido (TCLE). Visitamos as casas dos informantes para explicar os objetivos e procedimentos a serem adotados. Os interessados em participar foram convidados a assinar o TCLE, de acordo com a legislação em vigor na época (Resolução 466/12 do Conselho Nacional de Saúde). O presente estudo foi submetido e autorizado pelo Comitê de Ética em Pesquisa envolvendo Seres Humanos da Universidade Estadual da Bahia (Parecer $n^{\circ}$ 343.778), como parte do projeto de pesquisa "Conhecimento e diversidade de plantas nativas do Cerrado no Oeste da Bahia, Nordeste do Brasil".

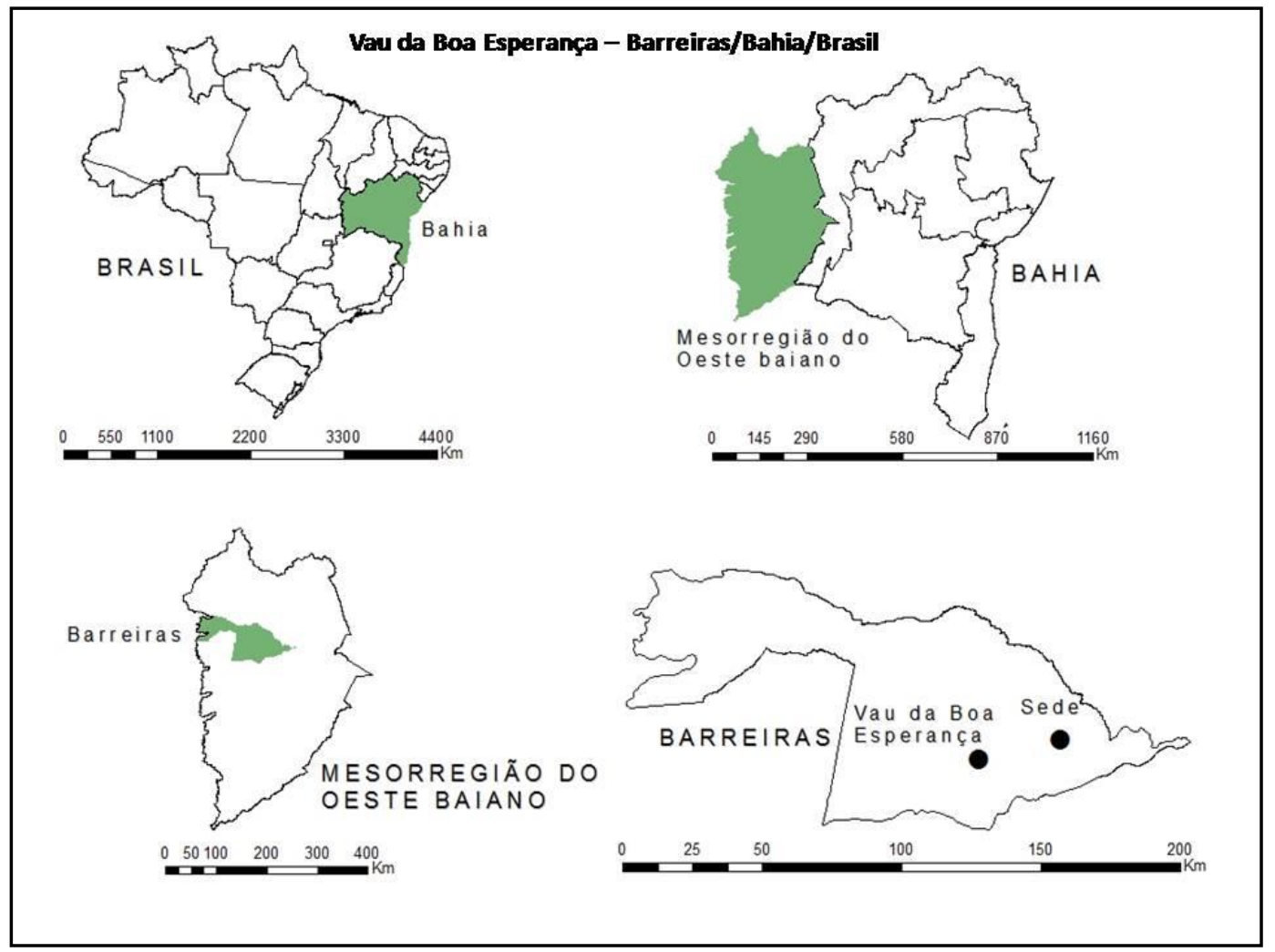

Figura 1: Mapa da comunidade Vau da Boa Esperança, município de Barreiras/BA (Fonte: P.M. Medeiros)

\section{Coleta e Análise de dados}

Conduzimos o estudo com vinte pessoas cuja faixa etária variou entre $22-83$. Utilizamos dez espécies alimentícias silvestres como modelo neste estudo (Tabela 1), que foram evidenciadas em um estudo prévio como aquelas comumente utilizadas como alimento pela população local e com maior frequência de consumo (Nascimento et al. 2015; Flora do Brasil 2020, em construção). No entanto, foram catalogadas na extensão de Cerrado que compreende a região cerca de 36 espécies alimentícias 
silvestres, utilizamos apenas as salientadas por Nascimento et al. (2015) por entendermos que o processo de coleta de dados seria exaustivo para os participantes, o que poderia ser um viés já que o foco deste estudo são dados qualitativos oriundos da representação de consumo das pessoas.

Utilizamos o termo "representação" para substituir o termo "percepção" uma vez que "percepção" se refere apenas àquilo que é percebido pelos sentidos fisiológicos (Gumuchian e Marois, 2000), havendo uma diferença do que é percebido e o que é expresso. Uma vez que as coisas no ambiente externo são percebidas pelos sentidos, dado que cada indivíduo acessa uma pequena representação da realidade (Silva et al. 2016) e consequentemente pode haver diferenças nas expressões dessa realidade, tanto de pessoa para pessoa quanto de ambiente para ambiente.

Assim, levando em consideração o perfil dos participantes utilizamos como ferramenta de coleta de dados um questionário semiestruturado (Albuquerque et al. 2014) composto por perguntas abertas e fechadas divididas em duas sessões; a primeira com o objetivo compreender os fatores que podem estar influenciando na permanência do consumo como também em sua diminuição; e o segundo com o intuito de identificar a frequência desse consumo e o sentimento que as pessoas possuem sobre elas.

Tabela 1: Dez espécies de plantas silvestres consumidas com maior frequência pelos moradores da comunidade Vau da Boa Esperança, Barreiras, Bahia, Nordeste do Brasil (Nascimento et al. 2015)

\begin{tabular}{lcc}
\hline Nome popular & Família & Espécie \\
\hline Araçá & Myrtaceae & Psidium sp. \\
Bacupari & Celastraceae & Salacia crassiflora Mart. \\
Buriti & Arecaceae & Mauritia flexuosa L.f \\
Bruto & Annonaceae & Annona crassiflora Mart. \\
Cagaita & Myrtaceae & Eugenia dysenterica DC. \\
Cajú & Anacardiaceae & Anacardium occidentale L. \\
Jatobá do campo & Fabaceae & Hymenaea stigonocarpa Mart. ex Hayne \\
Mangaba & Apocynaceae & Hancornia speciosa Gomes \\
Pequi & Caryocaraceae & Caryocar brasiliense Cambess \\
Puçá & Melastomataceae & Mouriri pusa Gardner \\
\hline
\end{tabular}

O questionário semiestruturado foi norteado pela seguinte questão em aberto: "O que $o(a)$ Senhor(a) acha de comer as plantas do mato?". Decidimos usar o termo "plantas do mato" porque, segundo Kinupp e Barros (2007), o termo está associado às espécies consideradas ervas daninhas, invasivas e selvagens, que muitas vezes são referidas pelas pessoas dessa maneira ou simplesmente "mato". Assim, era mais fácil para as pessoas entenderem a que estávamos nos referindo a plantas silvestres que não são adquiridas por meio de mercados ou feiras livres de grande demanda comercial, por exemplo, laranja, maçã, abacaxi, entre outras que não são encontradas em ambientes naturais e que não fazem parte da composição do ecossistema local. E para identificar a presença de fatores que influenciam ou limitam o consumo das espécies, fizemos os seguintes questionamentos: "Você acha que há coisas boas neles? Se sim, quais?" e "Você acha que há coisas ruins nelas? Se sim, quais?".

As respostas foram sistematizadas e associadas a 16 categorias, com base no estudo de Cruz et al. (2014), que apresenta características atribuídas a fatores positivos, ou seja, correspondentes às respostas que favoreceram o uso de alimentos das espécies estudadas, e fatores negativos, incluindo categorias formuladas através de respostas que inibem o uso de tais recursos, diagnosticando também espécies que foram relacionados a cada categoria. Foi estimado o percentual obtido das citações de cada categoria. Ainda no primeiro eixo da investigação utilizamos três questionamentos abertos para identificarmos um discurso padrão sobre os pensamentos e opiniões dos participantes sobre as plantas alimentícias silvestres: (1) "O que você acha de comer mato?"; (2) "O que fez você comer plantas arbustivas?"; (3) "Como você diria que o consumo dessas plantas é feito pelos jovens hoje em dia?".

As respostas foram analisadas por meio da Análise do Sujeito Coletivo (Lefevre \& Lefevre, 2005), que consiste em uma análise qualitativa das narrativas dos participantes afim de construir um ou 
mais discursos padrões e coerentes que representem um determinado pensamento ou representação social acerca de um fenômeno, preservando as dimensões individual e coletiva articulada (Lefevre \& Lefevre, 2014). O método utiliza a busca de ideias centrais ou palavras-chave utilizadas em todas as narrativas como expressões que correspondem a pensamentos e opiniões similares. Essas expressões podem ser trechos ou transcrições literais dos discursos que ao serem analisados representam o que pensam as coletividades (Lefevre \& Lefevre, 2005). Os discursos construídos foram analisados e divididos entre aqueles que apresentam argumentos sobre fatores que limitam o consumo das espécies alimentícias silvestres, e aqueles que narram elementos que influenciam a permanência do consumo.

\section{RESULTADOS E DISCUSSÃO}

\section{Representação sobre o consumo de plantas alimentícias silvestres}

A amostra estudada de 20 pessoas foi composta por indivíduos com idades acima de 46 anos, sendo dez mulheres e dez homens correspondendo a $83 \%$ do universo total. Dentro da amostra apenas duas pessoas apresentaram as idades mais juvenis (22 e 23 anos respectivamente). A partir da categorização das respostas dos participantes verificamos que das 16 categorias (Tabela 2), 11 foram consideradas positivas, ou seja, estimulam o consumo da espécie, encontrando um total de 125 citações. Entre elas, as categorias de mais representantes foram Topofilia 18,5\% das citações, Percepção de saúde ( $18,4 \%$ das citações) e Produto Natural (17\% das citações). Uma vez que houve uma quantidade de citações semelhantes para essas categorias, é possível que as três juntas contribuam para o estímulo do consumo dessas espécies.

Foram encontradas sete categorias que limitam o consumo dessas espécies, as quais receberam um total de 51 citações, dentre as quais se destacaram: Disponibilidade (45\% das citações), sendo associadas as respostas que consideraram o baixo número de indivíduos de determinadas espécies na área, um obstáculo ao seu consumo. Seguido de Substituição por produtos industrializados $(11,7 \%$ das citações) e Propriedades organolépticas (11,7\% das citações). Houve categorias que tiveram citações associados a aspectos positivos e negativos, como a Disponibilidade e as Propriedades Organolépticas nos quais a primeiro obteve $45 \%$ das citações como negativo e $11 \%$ como positivo, o segundo atingiu $10,4 \%$ nas citações como representação positiva e $11,7 \%$ como negativo.

Tabela 2: Categorias usadas como parâmetro para entender os fatores que influenciam o consumo das dez principais plantas alimentícias silvestres da comunidade Vau da Boa Esperança, Barreiras, Bahia, Nordeste do Brasil. Baseado em Cruz et al. (2014).

\begin{tabular}{|c|c|c|c|}
\hline CATEGORIA & DESCRIÇÃO & $\begin{array}{l}\text { PORCENTAGEM } \\
\text { DE CITAÇÃO }\end{array}$ & ESPÉCIES ATRIBUÍDAS \\
\hline \multicolumn{4}{|c|}{ Positivo } \\
\hline $\begin{array}{l}\text { Importância para a } \\
\text { comunidade }\end{array}$ & $\begin{array}{l}\text { Refere-se às respostas nas quais consideram o consumo dessas plantas importante para a } \\
\text { comunidade de alguma maneira desconhecida. }\end{array}$ & $2,4 \%$ & $\begin{array}{c}\text { Psidium sp. } \\
\text { M. flexuosa } \\
\text { H. stigonocarpa } \\
\text { C. brasiliense } \\
\text { M.pusa }\end{array}$ \\
\hline $\begin{array}{l}\text { Alimento } \\
\text { emergencial }\end{array}$ & Inclui aquelas plantas que são usadas para alimentar em tempos de fome. & $6,5 \%$ & $\begin{array}{l}\text { E. dysenterica } \\
\text { H. stigonocarpa } \\
\text { H. speciosa }\end{array}$ \\
\hline $\begin{array}{l}\text { Propriedades } \\
\text { organolépticas }\end{array}$ & $\begin{array}{l}\text { Agrupa respostas nas quais as pessoas consideram tópicos como aroma agradável ou } \\
\text { sabor como fatores positivos que favorecem o uso. }\end{array}$ & $10,4 \%$ & $\begin{array}{l}\text { Psidium sp. } \\
\text { S. crassiflora } \\
\text { M. flexuosa } \\
\text { A. crassiflora } \\
\text { E. dysenterica } \\
\text { A. occidentale } \\
\text { H. stigonocarpa } \\
\text { C. brasiliense } \\
\text { M. pusa }\end{array}$ \\
\hline Produto natural & $\begin{array}{l}\text { Agrupa as respostas que consideram as plantas como produtos naturais, sem a presença } \\
\text { de fertilizantes industriais e que não precisam de manejo e plantio. }\end{array}$ & $17 \%$ & $\begin{array}{l}\text { Psidium sp. } \\
\text { S. crassiflora } \\
\text { M. flexuosa } \\
\text { A. crassiflora, } \\
\text { E. dysenterica, } \\
\text { A. occidental } \\
\text { H. stigonocarpa } \\
\text { H. speciosa } \\
\text { C. brasiliense } \\
\text { M. pusa }\end{array}$ \\
\hline
\end{tabular}




\begin{tabular}{|c|c|c|c|}
\hline CATEGORIA & DESCRIÇÃO & $\begin{array}{l}\text { PORCENTAGEM } \\
\text { DE CITAÇÃO }\end{array}$ & ESPÉCIES ATRIBUÍDAS \\
\hline Percepção de saúde & $\begin{array}{l}\text { Reúne respostas que estimulam o uso de plantas devido às propriedades medicinais e } \\
\text { nutricionais. }\end{array}$ & $18,4 \%$ & $\begin{array}{c}\text { Psidium sp. } \\
\text { S. crassiflora } \\
\text { M. flexuosa } \\
\text { A. crassiflora } \\
\text { E. dysenterica } \\
\text { A. occidentale } \\
\text { C. brasiliense } \\
\text { M. pusa }\end{array}$ \\
\hline Topofilia* & $\begin{array}{l}\text { Categoria atribuída às respostas nas quais as pessoas enfatizam a importância do } \\
\text { ambiente natural, como também dos elementos que o constitui em que uma planta é } \\
\text { importante para a sobrevivência de outros indivíduos, para a conservação do meio } \\
\text { ambiente, para a beleza, para o que fornecem (sombra, ar fresco, comida etc.). }\end{array}$ & $18,5 \%$ & $\begin{array}{l}\text { Psidium sp. } \\
\text { S. crassiflora } \\
\text { E. dysenterica } \\
\quad \text { M. pusa }\end{array}$ \\
\hline Disponibilidade & $\begin{array}{l}\text { Categoria que se refere às respostas que abordam a quantidade de plantas encontradas no } \\
\text { local com base no presente e no passado, consideradas positivas, para um número maior } \\
\text { no momento presente. }\end{array}$ & $11 \%$ & $\begin{array}{l}\text { Psidium sp. } \\
\text { M. flexuosa }\end{array}$ \\
\hline Comercialização & $\begin{array}{l}\text { Refere-se a venda das espécies que foi considerada neste estudo como positiva, } \\
\text { considerando que a atividade gera renda para as pessoas. }\end{array}$ & $11 \%$ & $\begin{array}{l}\text { M. flexuosa, } \\
\text { H. stigonocarpa } \\
\text { C. brasiliense } \\
\quad \text { M. pusa }\end{array}$ \\
\hline $\begin{array}{l}\text { Exploração } \\
\text { madeireira }\end{array}$ & $\begin{array}{l}\text { Plantas que, além de terem seus frutos utilizados como alimento, sua madeira também é } \\
\text { utilizada na fabricação de móveis, lenha e/ou construção. }\end{array}$ & $0,8 \%$ & H. stigonocarpa \\
\hline $\begin{array}{l}\text { Disseminação do } \\
\text { conhecimento }\end{array}$ & $\begin{array}{l}\text { Reúne respostas nas quais é mencionado positivamente que deve o consumo destas } \\
\text { plantas deve ser apresentado a pessoas que não conhecem. }\end{array}$ & $5,8 \%$ & $\begin{array}{l}\text { Psidium } \mathrm{sp.}, \\
\text { M. flexuosa } \\
\text { H. stigonocarpa }\end{array}$ \\
\hline Afetivo & $\begin{array}{l}\text { Corresponde às respostas que mostram algum sentimento vindo do uso ou desuso das } \\
\text { plantas. }\end{array}$ & $4 \%$ & $\begin{array}{l}\text { Psidium sp. } \\
\text { E. dysenterica }\end{array}$ \\
\hline \multicolumn{4}{|c|}{ Negativo } \\
\hline Coleta & $\begin{array}{l}\text { Esta categoria inclui as respostas nas quais as pessoas mencionam a dificuldade de coleta } \\
\text { e o local de difícil acesso. }\end{array}$ & $8 \%$ & $\begin{array}{l}\text { M. pusa } \\
\text { S. crassiflora } \\
\text { M. flexuosa }\end{array}$ \\
\hline Indiferença & $\begin{array}{l}\text { Refere-se às respostas que mencionam de maneira negativa o desuso devido à presença } \\
\text { contínua das plantas, desencorajando as pessoas a utilizá-las. }\end{array}$ & $2 \%$ & $\begin{array}{l}\text { M. flexuosa } \\
\text { A. crassiflora } \\
\text { E. desinterica } \\
\quad \text { M. pusa }\end{array}$ \\
\hline Incentivo & $\begin{array}{l}\text { Agrupa respostas nas quais a falta de incentivo causa uma diminuição no consumo } \\
\text { alimentar das espécies. }\end{array}$ & $5,8 \%$ & $\begin{array}{l}\text { Psidium sp. } \\
\text { M. flexuosa } \\
\text { A. crassiflora } \\
\text { A. occidentale } \\
\text { E. dysenterica } \\
\text { H. stigonocarpa }\end{array}$ \\
\hline Outros & $\begin{array}{l}\text { Agrupa as respostas mencionadas por um pequeno número de pessoas que não pôde ser } \\
\text { incluído nas outras categorias criadas. }\end{array}$ & $10 \%$ & $\begin{array}{l}\text { S. crassiflora } \\
\text { C. brasiliense }\end{array}$ \\
\hline $\begin{array}{l}\text { Substituição por } \\
\text { industrializados }\end{array}$ & $\begin{array}{l}\text { Categoria que reúne respostas que mencionam o desuso de plantas pela introdução de } \\
\text { produtos industrializados. }\end{array}$ & $11,7 \%$ & $\begin{array}{l}\text { Psidium sp. } \\
\text { S. crassiflora } \\
\text { A. crassiflora } \\
\text { M. flexuosa } \\
\text { H. stigonocarpa } \\
\text { C. brasiliense } \\
\text { M. pusa }\end{array}$ \\
\hline Disponibilidade & $\begin{array}{l}\text { Categoria refere-se às respostas que abordam a quantidade de plantas encontradas no } \\
\text { local no presente e em relação ao passado, consideradas negativas se esse número for } \\
\text { pequeno. }\end{array}$ & $45 \%$ & $\begin{array}{l}\text { Psidium sp. } \\
\text { S. crassiflora } \\
\text { M. flexuosa } \\
\text { A. occidentale } \\
\text { H. stigonocarpa } \\
\text { H. speciosa } \\
\text { C. brasiliense } \\
\text { M. pusa }\end{array}$ \\
\hline $\begin{array}{l}\text { Propriedades } \\
\text { organolépticas }\end{array}$ & $\begin{array}{l}\text { Agrupa respostas nas quais as pessoas consideram questões como aroma desagradável } \\
\text { ou sabor como limitantes para o consumo. }\end{array}$ & $11,7 \%$ & $\begin{array}{l}\text { Psidium sp. } \\
\text { S. crassiflora } \\
\text { M. flexuosa } \\
\text { A. crassiflora } \\
\text { E. dysenterica } \\
\text { H. stigonocarpa } \\
\text { H. speciosa } \\
\text { C. brasiliense } \\
\end{array}$ \\
\hline
\end{tabular}


Ao sistematizar as respostas para as perguntas: "Você acha que há coisas boas nelas? Em caso afirmativo, quais são elas?" e "Você acha que existem coisas ruins? Em caso afirmativo, quais?", foi possível atribuir as categorias de representação encontradas para cada espécie estudada. De acordo com a Tabela 2, dentre as categorias de aspectos positivos (aqueles que promovem o uso de alimentos), a categoria Produto Natural foi a representação atribuída a todas as dez espécies, seguida de Propriedades Organolépticas atribuídas a nove espécies e Percepção de Saúde, oito das dez espécies estudadas. Entre as categorias negativas, a que apresentou o maior número de espécies vegetais relacionadas foi a propriedade Organoléptica, uma representação atribuída a um total de nove espécies, seguida pela Disponibilidade, associada a oito espécies e Substituição por produtos industriais por sete espécies.

\section{Análise do Sujeito Coletivo}

A partir das respostas fornecidas pelos entrevistados, as perguntas abertas: "O que você acha de comer mato? E o que você ach a deles?", "Qual é o motivo que o levou a experimentar essas plantas?" e "Como você diria que o consumo dessas plantas é feito pelos jovens hoje em dia?", dez ideias centrais foram identificadas por meio de expressões-chave, as quais receberam as categorias de representações estabelecidas para a análise dos dados. No entanto, percebeu-se que mais de uma representação estava associada a cada questão e, devido às inúmeras linhas correspondentes às mesmas expressões-chave, foi preferível deixar o apresentar aqui apenas os discursos referentes as categorias que obtiveram maiores citações.

Assim, foi possível diagnosticar seis representações positivas sobre o consumo das espécies e seu respectivo número de citações em ordem crescente: Produto Natural (60\%), Percepção de saúde (30\%), Alimento emergencial (20\%), Comercialização (10\%), Topofilia (10\%) e Tecnologia (5\%), permitindo a construção do seguinte discurso do sujeito:

"É natural, é bom! São frutas que vêm da natureza, sem veneno e com vitaminas, sabemos de onde vêm ... São boas pra saúde, são boas porque não têm veneno, são muito saudáveis [...] Muito bom pra quando não há nada pra comer em casa, porque são naturais, importantes para a alimentação na época de seca, matam a fome e nutrem na época em que não há nada ... Além de comer vendemos, ganhamos dinheiro extra [...] Devem ser preservadas, são importantes pra outros animais [...] Você pode fazer móveis e ferramentas além de comer ".

Neste primeiro discurso é nítido a percepção das pessoas sobre o potencial terapêutico que as espécies possuem, no entanto, a principal ideia trazida pelo discurso é o fato das espécies serem naturais e não apresentarem "veneno", ou seja, não possuírem nenhuma interferência de produtos químicos no seu processo de plantio. Segundo Ditlevsen et al. (2019) a pureza presente nos alimentos é um dos principais fatores de avaliação de qualidade. Assim, a ausência de aditivos alimentares, conservantes ou resíduos traz a ideia de pureza e naturalidade aos alimentos, associados ao conceito de uma natureza imaculada e intocável que, frequentemente, é vista como a equivalência de algo puro. Nesse sentido, a pureza do alimento reflete o quão saudável ele é (Ditlevsen et al. 2019).

Além disso, para os informantes, as representações positivas Disseminação e Afetivo foram as mais expressivas sobre a influência do consumo das espécies pela primeira vez, obtendo $70 \%$ e $15 \%$ nas citações, fazendo o discurso:

"Os mais velhos nos contavam histórias sobre essas plantas [...] nossos pais que ensinavam, os mais velhos nos davam [...] eu comi quando criança porque me disseram que era bom [...] meu pai trouxe do mato e me deu [...] Brincando no mato, eu via e comia, porque eu achava bonito, lá na minha infância, quando tudo estava bem, eu morava no mato, encontrava e comia, porque sabia que era Deus quem deixou ali".

As primeiras experimentações das espécies podem estar associadas a eventos nos quais os participantes quando crianças iam até fragmentos florestais ou por influência de adultos que já consumiam as espécies. No entanto, a influência de pessoas mais velhas é extremamente importante no processo de aprendizagem, pois as preferências alimentares são influenciadas pelo condicionamento 
associativo que permite os indivíduos aprenderem que o consumo de certos alimentos acompanha consequências positivas ou negativas (Contento, 2007). No caso das crianças, Landry et al. (2019) argumenta que novos alimentos são mais difíceis de serem aceitos devido ao seu sabor, textura e muitas vezes aparência, mas podem ser eventualmente apreciados e aceitos com uma exposição repetitiva ou à segurança e familiaridade trazida por esse evento. Logo, a influência de uma pessoa de idade mais avançada, que muitas vezes faz parte do ciclo familiar reforça o sentimento de segurança, facilitando a apreciação desse novo alimento.

Ao questionar sobre o consumo de espécies silvestres pelos jovens atualmente, obteve-se o seguinte discurso:

"Os pais não estão interessados em trazer, muitos crescem e nem conhecem essas frutas, devemos incentivar [...] hoje eles só querem coisas industrializadas, comem apenas as coisas da rua, [...] tem quase não mais, os jovens não comem porque não veem essas frutas como a gente via, essas frutas já são escassas, muitos crescem e não sabem ".

Através do discurso acima, podem-se identificar representações associadas à limitação no consumo das espécies, sendo a Substituição por produtos industrializados a que obteve maior representatividade em cerca de $40 \%$ das citações, seguida pela Disponibilidade $30 \%$ e a falta de Incentivo $20 \%$ em citações.

No entanto, uma pequena parte dos informantes argumentou que atualmente os jovens ainda consomem a espécie, sendo representações positivas como fatores associados ao consumo, as categorias Disponibilidade e Difusão de conhecimento, ambas obtendo apenas $5 \%$ nas citações, quanto às falas:

"Consumo mais do que antes, e eles passam para a próxima geração [...] Hoje, essas frutas são mais fáceis de encontrar, existem por aqui."

Além disso, questionou-se se a falta dessas espécies afetaria de alguma forma a vida dos informantes, e através de suas respostas foram encontradas 4 categorias de representações recebendo um total de 25 citações, distribuídas entre: Topofilia (50\%), Comercialização (45 \%), Percepção de saúde $(20 \%)$ e afetivo $(10 \%)$, podendo construir o seguinte discurso:

"Os animais do mato sentiriam falta deles, reduziriam a comida para todos os animais e afetariam nossas vidas porque nós os comemos, isso afetaria porque queremos ver eles na natureza, mesmo que a gente não coma, tudo tem sua função e eles mantêm o ambiente bonito, a falta deles prejudicaria o meio ambiente e a gente [...] Seria ruim porque, além de comer, existem pessoas que vivem da venda dessas frutas e das coisas que delas produzem. , a gente vende e esse dinheiro ajuda na casa, tem gente que mora nela, gera renda ... Afetaria a gente comer, seria horrivel porque além de comer é bom pra saúde, é remédio [. ..] Sentiríamos falta, já estamos acostumados a comer eles."

O sentimento presente no discurso acima retrata uma ligação positiva entre as pessoas e as plantas, e a importância delas para o ambiente. Permitindo observar facetas de bem-estar além de satisfação com à conexão com a natureza. Howell et al. (2011) afirma que esse sentimento de conexão está correlacionado positivamente com o bem-estar psicológico, emocional e social, envolvendo uma sensação de relacionamento com algo maior que si mesmo. Por meio das representações dos moradores tanto das categorias pré-estabelecidas e pelas falas é possível detectar que eles têm um ponto de vista positivo sobre o consumo alimentar das espécies silvestres do Cerrado, ao afirmar que são importantes na manutenção e equilíbrio do meio ambiente, bem como em suas vidas. Esse resultado foi evidenciado de acordo com a representatividade da categoria Topofilia, que é contra o que Tuan (1980) traz à percepção, atitude e valores que atribuímos ao meio ambiente, em seu estudo ele define o termo Topofilia como laços afetivos entre seres humanos e o meio ambiente, diferindo profundamente em intensidade, sutileza e forma de expressão, afirmando que topofilia é o sentimento que nos faz sentir o meio ambiente, de maneira íntima e afetiva.

Segundo Kellert (1970), essas associações têm um caráter evolutivo e adaptativo, no qual a 
percepção naturalista reflete dois níveis, um simples que demonstra satisfação no contato com os recursos naturais e um nível mais complexo que associa um fascínio e um certo curiosidade em explorálo. Além de uma percepção humanística, reflete sentimentos de afeição por elementos ambientais individuais, como o cuidado e a preservação de certas espécies vegetais e animais. Essa análise é semelhante à encontrada por Ladio (2001), na qual a autora propôs identificar os motivos responsáveis pela preservação das plantas alimentícias silvestres presentes em uma comunidade Mapuche na Patagônia, evidenciando que as pessoas têm a necessidade de consumir fontes alternativas de alimentos, ou seja, recursos naturais que não receberam nenhum tipo de intervenção humana, como manuseio e pesticidas, para tentar preservá-los no meio ambiente.

A percepção de que as espécies são saudáveis e naturais, por isso continuam fazendo parte da dieta local traz evidências diferentes do que tem sido retratado na literatura (Grosskinsky e Gullick 2000). Diversos estudos têm demostrado o abandono do consumo de espécies silvestres devido o aumento da inserção de produtos industrializados e frutos cultivados. Nascimento et al. (2014), argumentam que a diminuição do consumo de espécies silvestres em regiões de floresta seca está relacionada ao estigma social, no qual seu consumo é socialmente considerado um símbolo de pobreza, associado a alimentos consumidos por pessoas com baixo poder aquisitivo. Essa contradição evidencia que o comportamento alimentar humano apresenta influências de sensações afetivas, como foi retratado nos discursos. Demonstrando que não nos alimentamos apenas para saciar as necessidades nutricionais. Em contra partida o ato de alimentar-se envolve emoções, e essas emoções podem contribuir para a manutenção e permanência de certas espécies na dieta local.

Contudo, a disponibilidade dos recursos é percebida pelas participantes como um fator que pode limitar o consumo das espécies. Berilli et al. (2007), mostram que existem poucos estudos sobre o crescimento e desenvolvimento de populações de espécies silvestres no Cerrado, dificultando o estabelecimento de uma base mais precisa que possa afirmar que há de fato uma baixa disponibilidade dessas espécies na vegetação. No entanto, o discurso dos informantes nos permite identificar que a diminuição e a possível ausência desses recursos podem afetar suas vidas. Esse resultado pode estar associado ao fato de o uso de alimentos fazer parte das práticas de subsistência de muitas populações humanas, principalmente nas áreas rurais e essa diminuição pode gerar implicações nutricionais, uma vez esses recursos fornecem micronutrientes, como vitamina A, ferro, folato, niacina e cálcio (Ickowitz et al. 2016). De acordo com Arnold et al. (2011) os vegetais silvestres não fazem parte dos alimentos básicos na nutrição diária como o milho, por exemplo, ou compõem a maioria da dieta; ao contrário, eles complementam o que já está disponível pela produção agrícola e pelos industrializados, aumentando a qualidade nutricional ao passo que aumenta a variedade alimentar (Hall et al. 2019), contribuindo substancialmente para a segurança alimentar de populações, principalmente, em áreas rurais.

\section{CONSIDERAÇÕES FINAIS}

Os resultados aqui apresentados permitem concluir que as percepções das pessoas sobre os recursos atuam fortemente em seus padrões de uso, sendo bons preditores para avaliar o uso de espécies alimentícias silvestres e, mesmo que outros fatores ecológicos e biológicos tenham sido identificados, a percepção de disponibilidade e a afetividade que as pessoas possuem com as espécies são cruciais para a manutenção da dieta local. O apego foi crucial no consumo das espécies, revelando que características intrínsecas do comportamento humano podem estar atuando na seleção de recursos. Assim, o estudo das representações torna-se uma ferramenta válida para acessar os fatores subjacentes às preferências locais e identificar possíveis padrões de consumo.

\section{AGRADECIMENTOS}

As autoras agradecem em primeiro lugar aos moradores da comunidade Vau da Boa Esperança pela receptividade e confiança oferecida. A Fundação de Amparo à Pesquisa do Estado da Bahia (FAPESB) pelo financiamento concedido à primeira autora e a Universidade do Estado da Bahia pelo apoio logístico.

\section{REFERÊNCIAS BIBILIOGRÁFICAS}

AlBUQUeRQUE, U. P.; RAMOS, M. A.; LUCENA, R. F. P.; ALENCAR, N. L. (2014) Methods and Techniques used to collect Ethnobiological. In: Albuquerque UP, Cunha LVFC, Lucena RFP, Alves RRNA (Eds), Methods and Techniques in Ethnobiology and Ethnoecology. 1ed. Springer, Switzerland, pp.15-38. 
Hora et al. "É natural, é bom! são frutos que vem da natureza": representações locais sobre o consumo de plantas alimentícias silvestres em uma área rural do Brasil Ethnoscientia 5, 2020. D.O.I.: 10.22276/ethnoscientia.v5il.286

GUIMARÃES, M.; MIRANDA, E. E.; VIEIRA, H. R.; VALLADARES, G. S.; MANGABEIRA, J. A. M. C. 2002. Monitoramento da expansão agropecuária na Região Oeste da Bahia. Embrapa Monitoramento por SatéliteDocumentos (INFOTECA-E).

BHARUCHA, Z.; PRETTY, J. 2010. The roles and values of wild foods in agricultural systems. Philosophical Transactions of the Royal Society B: Biological Sciences, 365: 2913-2926.

BERILI, S. S.; OLIVEIRA, J. G.; MARINHO, A. B.; LYRA, G. B.; SOUSA, E. F.; VIANA, A. A. P.; BERNARDO, S.; PEREIRA, M. G. 2007. Avaliação da taxa de crescimento de frutos de mamão (Carica papaya L.) em função das épocas do ano e graus-dias acumulados. Revista Brasileira de Fruticultura, 29: 11-14.

CAMPOS, L. Z. O.; ALBUQUERQUE, U. P.; PERONI, N.; ARAÚJO, E. L. 2015. Do socioeconomic characteristics explain the knowledge and use of native food plants in semiarid environments in Noetheastern Brazil? Journal of Arid Environments, 115: 53-61.

COTTON, C. M. (1996). Ethnobotany: Principles and applications. Chichester, England: John Wiley and Sons, pp. 434.

CONTENTO, I. R. (2007). Nutrition education: linking research, theory, and practice. Jones \& Bartlett Learning.

CRUZ, M. P.; MEDEIROS, P. M.; SARMIENTO-COMBARIZA, I.; PERONI, N.; ALBUQUERQUE, U. P. 2014. "I eat the manofê so it is not forgotten": local perceptions and consumption of native wild edible plants seasonal dry forests in Brazil. Journal of Ethnobiology and Ethnomedicine, 10-45.

DITLEVSEN, K.; SANDOE, P.; LASSEN, J. 2019. Healthy food is nutritious, but organic food is healthy because it is pure: The negotiation of healthy food choices by Danish consumers of organic food. Food Quality and Preference, 71: 46-53.

Flora do Brasil 2020 em construção. Jardim Botânico do Rio de Janeiro. Disponível em: <http://floradobrasil.jbrj.gov.br/reflora/floradobrasil/FB10388>. Acesso em: 27 abr. 2020

NASCIMENTO, V. T.; DE MOURA, N. P.; DA SILVA VASCONCELOS, M. A.; MACIEL, M. I. S.; DE ALBUQUERQUE, U. P. (2011) Chemical characterization of native wild plants of dry seasonal forests of the semi-arid region of northeastern Brazil. Food Research International 44:2112-2119

GROSSKINSKY, B.; GULLICK, C. 2000. Exploring the Potential of Indigenous Wild Food Plants in Southern Sudan: Proceedings of a workshop held in Lokichoggio, Kenya, June 3- 5 1999. Prepared by The Mitchell Group, 139.

HALL, C.; MACDIARMID, J. I.; MATTHEWS, R. B.; SMITH, P.; HUBBARD, S. F.; DAWSON, T. P. (2019) The relationship between forest cover and diet quality: a case study of rural southern Malawi. Food Security 11:635-650

HOWELL, A. J.; DOKPO, R. L.; PASSMORE, H. A.; BURO, K. 2011. Nature connectedness: Associations with well-being and mindfulness. Personality and Individual Differences, 51: 166-171.

Instituto Brasileiro de Estatística e Geografia. Panorama do município de Barreiras, estado da Bahia. Disponível em: <https://cidades.ibge.gov.br/brasil/ba/barreiras/panorama>. Acessado em: 02 de maio de 2020.

ICKOWITZ, A.; ROWLAND, D.; POWELL, B.; SALIM, M. A.; SUNDERLAND, T. (2016). Forests, trees, and micronutrient-rich food consumption in Indonesia. PLoS One, 11(5), e0154139

KINUPP, V. F.; BARROS, I. B. I. 2007. Riqueza de plantas alimentícias não-convencionais na Região Metropolitana de Porto Alegre, Rio Grande do Sul. Revista Brasileira de Biociências, 5: 63-65.

KUHNLEIN, H. V.; RECEVEUR, O. (1996) Dietary Change and Traditional Food Systems of Indigenous Peoples. Annual Review of Nutrition 16:417-442

LADIO, A. 2001. The maintenace of wild edible plant gathering in a Mapuche Community of Patagonia. Economic Botany, 55: 243-254

LADIO, A. H.; LOZADA, M. 2004. Patterns of use and knowledge of wild edible plants in distinct ecological environments: a case study of a Mapuche community from northwestern Patagonia. Biodiversity and Conservation, 13: 1153-1173.

LADIO, A. H.; LOZADA, M.; WEIGANDT, M. 2007. Comparison of traditional wild plants use between two Mapuche communities inhabiting arid and forest environments in Patagonia, Argentina. Journal of Arid Environments, 69: 695-715.

LANDRY, M.; LEMIEUX, S.; LAPOINTE, A.; BÉDARD, A.; BÉLANGER-GRAVEL, A.; BÉGIN, C.; PROVENCHER, Vé.; DESROCHES, S. 2018. Is eating pleasure compatible with healthy eating? A qualitative study on Quebecers' perceptions, Appetite, doi: 10.1016/j.appet.2018.02.033.

LEFÈVRE, F.; LEFÈVRE, A. M. C. 2005. Depoimentos e Discursos. Uma nova Proposta de Análise em Pesquisa Social. Série Pesquisa. Vol. 12. Líber Livro, Brasília, DF, Brasil.

NASCIMENT, V. T.; DE MOURA, N. P.; VASCONCELOS, M. A. S.; MACIEL, M. I. S.; ALBUQUERQUE, U. P. 2011. Chemical characterization of native wild plants of dry seasonal forests of the semiarid region of northeastern Brazil. Food Research International, 44: 2112-2119.

NASCIMENTO, V. T.; PEREIRA, H. C.; SILVA, A. S.; FlORENTINO, A. T. N.; MEDEIROS, P. M. 2015. Plantas alimentícias não convencionais conhecidas por uma comunidade rural do cerrado, no município de Barreiras, Oeste da Bahia. Revista Ouricuri, 5: 86-109. 
NYMBAYO, I. 2015. Food Security In Developed Countries (Europe and USA) - Is it Insecurity and Insufficiency or Hunger and Poverty in Developed Countries? BAOJ Nutrition, 1: 1-7.

NUNES, E. N.; GUEERA, N. M.; ARÉVALO-MARÍN, E.; ALVES, C. A. B.; NASCIMENTO, V. T.; CRUZ, D. D.; LADIO, A. H.; SILVA, S. M.; OLIVEIRA, R. S.; LUCENA, R. F. P. 2018. Local botanical knowledge of native food plants in the semiarid region of Brazil. Journal of Ethnobiology and Ethnomedicine, 14: 1-13.

PEEL, M. C.; FINLAYSON, B. L.; MCMAHON, T. A. 2007. Updated world map of the Koppen-Geiger climate classification. Hydrology and Earth System Sciences, 11: 1633-1644.

SATTERTHWAITE, D.; MCGRANAHAN, G.; TACOLI, C. 2010. Urbanization and its implications for food and farming. Philos Trans R Soc Lond B Biol Sci, 27: 2809-2820.

SOLIVA, R.; HUNZIKER, M. 2009. Beyond the visual dimension: Using ideal type narratives to analyse people's assesments of landscape scenarios. Land Use Policy, 26, 284-294.

TUAN, Y. 1980. Topofilia: um estudo da percepção, atitudes e valores do meio ambiente. Difel, Difusão editorial S.A. São Paulo, SP, Brasil, 228p.

WILSON, E. O. 1984. Biofilia. Fondo de Cultura Económica. México, 283 